\title{
Ferromagnetism in $\mathrm{UGe}_{2}: \mathrm{A}$ microscopic model
}

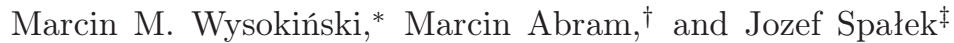 \\ Instytut Fizyki im. Mariana Smoluchowskiego, Universytet Jagielloński, Reymonta 4, PL-30-059 Kraków, Poland
}

(Dated: November 8, 2018)

\begin{abstract}
Anderson lattice model is used to rationalize the principal features of the heavy fermion compound $\mathrm{UGe}_{2}$ by means of the generalized Gutzwiller approach (the SGA method). This microscopic approach successfully reproduces magnetic and electronic properties of this material, in a qualitative agreement with experimental findings from the magnetization measurements, the neutron scattering, and the de Haas-van Alphen oscillations. Most importantly, it explains the appearance, sequence, character, and evolution in an applied magnetic field of the observed in $\mathrm{UGe}_{2}$ ferro- and, para-magnetic phases as an effect of a competition between the $f-f$ electrons Coulomb interaction energy and $f$-conduction electrons kinetic energy (hybridization).

PACS numbers: 71.27.+a,75.30.Kz,71.10.-w
\end{abstract}

Introduction. The discovery of the spin-triplet superconductivity (SC) inside the ferromagnetic (FM) phase of heavy-fermion compound $\mathrm{UGe}_{2}{ }^{1}$ sparked an intense discussion about the cause of such coexistence. Although the spin-triplet paired phase has been known to appear in the condensed ${ }^{3} \mathrm{He}{ }^{2}$ and most likely in $\mathrm{Sr}_{2} \mathrm{RuO}_{4} \stackrel{3}{ }$, until its discovery in $\mathrm{UGe}_{2}$ there was no convincing example for a strongly FM material hosting SC.

Specifically, the phase diagram for $\mathrm{UGe}_{2}$ on the temperature-pressure $(\mathrm{T}-\mathrm{p})$ plane contains both SC and two FM phases, with stronger and weaker magnetization ${ }^{4}$, usually referred to as FM2 and FM1, respectively, as well as paramagnetic phase (PM), with the phase transitions between them of the 1st order for low temperature, $T \lesssim 7 K \underline{\underline{5}}$. The FM-SC coexistence is strongly suggestive of a single mechanism based on magnetic correlations which is responsible for both FM and $\mathrm{SC}$ appearance and thus should be treated on equal footing, as e.g. in $\mathrm{UGe}_{2}$ both phases disappear at the same pressure 1.4 . Another indication of the coupled nature of both phases is that $\mathrm{SC}$ dome on the $\mathrm{T}-\mathrm{p}$ plane coincides with the phase transition between FM2 and FM1 1 . Thus, we address here in detail the question of microscopic origin of the observed ferromagnetism, as it should bring us closer to determining the mechanism of superconductivity. The question related to the inclusion of SC requires a separate study $\underline{\underline{6} .7}$ (see discussion at the end).

Experimental observations suggest that the ferromagnetism in $\mathrm{UGe}_{2}$ has an itinerant nature $\frac{1,8,9}{2}$ and is mediated by the uranium $5 f$ electrons $1,10,11$. Delocalization of the $5 f$ electrons can be interpreted as resulting from hybridization of $5 f$ originally atomic states with those from conduction band $\stackrel{8}{\underline{\varepsilon}}$ derived from $p$ states due to Ge and $d-s$ states due to U. This is supported by a noticeable difference of the effective paramagnetic moment per uranium atom in this compound with respect to the corresponding atomic value for either $f^{3}$ or $f^{2}$ configurations ${ }^{\underline{1}}$, as well as from a fractional value of the magnetization relative to the moment saturation. This means that the Hund's rule coupling in the atomic sense is broken, and the itineracy of $5 f$ electrons is the source of the band ferromagnetism in which Hund's ferromagnetic interaction plays a role in combination with the stronger intra-atomic Coulomb interaction. This also means that the $f$-electron orbital degeneracy is not essential, but the role of the hybridization is.

Apart from other theories concerning origin of FM in considered class of materials $\frac{12,13}{13}$ there exists $\frac{14}{14}$ a phenomenological rationalization of the magnetic properties within a rigid-band Stoner approach, which requires introduction of an ad hoc two-peaked structure of density of states (DOS) near the Fermi surface (FS). Our purpose is to invoke a microscopic description starting from the Anderson-lattice model (ALM) which is appropriately adapted to the heavy-fermion compound $\mathrm{UGe}_{2}$. This comprises a relatively simple quasi-two-dimensional electronic structure ${ }^{15-17}$. From such starting point an effective non-rigid two-band description arises naturally and allows for a detailed rationalization of magnetic and electronic properties, at least on a semiquantitative level. Additionally, as the correlations among $5 f$ electrons are sizable, an emergence of the Stoner-like picture of FM can be accounted for only with inclusion specific features coming from the electronic correlations. Although the resulting rationalization of the physical properties is semiquantitative in nature, it provides in our view a coherent picture of a number of properties $4,10,11,18,19$.

Model. We solve ALM by means of a variational treatment with the Gutzwiller wave function, $\left|\psi_{G}\right\rangle=\prod_{\mathrm{i}} \hat{P}_{\mathbf{i}}\left|\psi_{0}\right\rangle$, where $\hat{P}_{\mathbf{i}}$ is the operator projecting out part of double occupancies from the uncorrelated groundstate $\left|\psi_{0}\right\rangle$ at site i. We have extended the standard approach $\underline{20}-22$ to the statistically consistent form 23 (SGA method). Explicitly, we start with the ALM Hamiltonian, with an applied magnetic field introduced via the Zeeman term $\left(h \equiv \frac{1}{2} g \mu_{B} H\right)$, i.e.,

$$
\begin{aligned}
\hat{\mathcal{H}}-\mu \hat{N} & =\sum_{\mathbf{i}, \mathbf{j}, \sigma}^{\prime} t_{\mathbf{i j}} \hat{c}_{\mathbf{i}, \sigma}^{\dagger} \hat{c}_{\mathbf{j}, \sigma}-\sum_{\mathbf{i}, \sigma}(\mu+\sigma h) \hat{n}_{\mathbf{i}, \sigma}^{c} \\
& +\sum_{\mathbf{i}, \sigma}\left(\epsilon_{f}-\mu-\sigma h\right) \hat{n}_{\mathbf{i}, \sigma}^{f}+U \sum_{\mathbf{i}} \hat{n}_{\mathbf{i}, \uparrow}^{f} \hat{n}_{\mathbf{i}, \downarrow}^{f} \\
& +V \sum_{\mathbf{i}, \sigma}\left(\hat{f}_{\mathbf{i}, \sigma}^{\dagger} \hat{c}_{\mathbf{i}, \sigma}+\hat{c}_{\mathbf{i}, \sigma}^{\dagger} \hat{f}_{\mathbf{i}, \sigma}\right)
\end{aligned}
$$


where primed sum denotes summation over all lattice sites $\mathbf{i} \neq \mathbf{j}, \hat{f}$ and $\hat{c}$ are operators related to $f$ - and $c$ orbitals respectively, with spin $\sigma=\uparrow, \downarrow$. We have also defined the total number of electrons operator as $\hat{N}$, and for the respective orbitals and spins as $\hat{n}_{\mathbf{i}, \sigma}^{f} \equiv \hat{f}_{\mathbf{i}, \sigma}^{\dagger} \hat{f}_{\mathbf{i}, \sigma}$, $\hat{n}_{\mathbf{i}, \sigma}^{c} \equiv \hat{c}_{\mathbf{i}, \sigma}^{\dagger} \hat{c}_{\mathbf{i}, \sigma}$. In our model, we consider finite intra- $f$ orbital Coulomb interaction $U$, the on-site inter-orbital hybridization $V<0$, the hopping amplitude between the first $(t)$, and the second $\left(t^{\prime}=0.25|t|\right)$, nearest neighboring sites, and the atomic level for $f$-states placed at $\epsilon_{f}=-3|t|$. In the following $|t|$ is used as energy unit.

First, we would like to evaluate the ground-state energy, $E_{G} \equiv\left\langle\psi_{G}|\hat{\mathcal{H}}| \psi_{G}\right\rangle /\left\langle\psi_{G} \mid \psi_{G}\right\rangle$. Applying the usual procedure ${ }^{21,22}$, called Gutzwiller approximation (GA), we simplify the projection to the local sites on which the operators from (1) act. In that manner one obtains the effective single-particle Hamiltonian in a momentum space with renormalized hybridization by the Gutzwiller narrowing factor $q_{\sigma}{ }^{20}$, namely

$$
\hat{\mathcal{H}}_{G A} \equiv \sum_{\mathbf{k}, \sigma} \Psi^{\dagger}\left(\begin{array}{cc}
\epsilon_{\mathbf{k}}^{c}-\sigma h-\mu & \sqrt{q_{\sigma}} V \\
\sqrt{q_{\sigma}} V & \epsilon_{f}-\sigma h-\mu
\end{array}\right) \Psi+\Lambda U d^{2},
$$

where we have defined $\Psi^{\dagger} \equiv\left(\hat{c}_{\mathbf{k}, \sigma}^{\dagger}, \hat{f}_{\mathbf{k}, \sigma}^{\dagger}\right), \Lambda$ denotes number of lattice sites, and $d^{2}$ is the probability of having doubly occupied $f$-orbital that we optimize variationally. In order to ensure that variationally calculated polarization and the $f$-level occupancy would coincide with those coming from the self-consistent procedure ${ }^{23}$, we modify our effective Hamiltonian (2) by introducing additional constraints on the polarization $\left(m_{f}\right)$ and the number $\left(n_{f}\right)$ of $f$-electron states via the Lagrange-multiplier method. The effective Hamiltonian with the constraints takes now the form

$$
\begin{aligned}
& \hat{\mathcal{H}}_{S G A} \equiv \\
& \hat{\mathcal{H}}_{G A}-\lambda_{n}^{f}\left(\sum_{\mathbf{k}, \sigma} \hat{n}_{\mathbf{k}, \sigma}^{f}-\Lambda n_{f}\right)-\lambda_{m}^{f}\left(\sum_{\mathbf{k}, \sigma} \sigma \hat{n}_{\mathbf{k}, \sigma}^{f}-\Lambda m_{f}\right) \\
& =\sum_{\mathbf{k}, \sigma} \Psi^{\dagger}\left(\begin{array}{rr}
\epsilon_{\mathbf{k}}^{c}-\sigma h-\mu & \sqrt{q_{\sigma}} V \\
\sqrt{q_{\sigma}} V & \epsilon_{f}-\sigma\left(h+\lambda_{m}^{f}\right)-\lambda_{n}^{f}-\mu
\end{array}\right) \Psi \\
& \\
& +\Lambda\left(U d^{2}+\lambda_{n}^{f} n_{f}+\lambda_{m}^{f} m_{f}\right) .
\end{aligned}
$$

Those constraint parameters $\lambda_{n}^{f}$ and $\lambda_{m}^{f}$ are also determined variationally. They play a role of nonlinear selfconsistent fields acting on the charge and the spin degrees of freedom respectively. Diagonalization of (3) in this spatially homogeneous case leads to four branches of eigenenergies, $E_{\mathbf{k} \sigma}^{ \pm}$representing two spin-split hybridized bands $E^{ \pm}$. In order to determine the equilibrium properties of the system, we need to find the minimum of the generalized Landau grand-potential functional $\mathcal{F}$,

$$
\begin{aligned}
\frac{\mathcal{F}}{\Lambda}= & -\frac{1}{\Lambda \beta} \sum_{\mathbf{k} \sigma b} \ln \left[1+e^{-\beta E_{\mathbf{k} \sigma}^{b}}\right] \\
& +\left(\lambda_{n}^{f} n_{f}+\lambda_{m}^{f} m_{f}+U d^{2}\right),
\end{aligned}
$$

where $b= \pm$. Effectively, it leads to the set of five nonlinear equations, $\frac{\partial \mathcal{F}}{\partial \vec{\lambda}}=0$ for $\vec{\lambda} \equiv\left\{d, n_{f}, m_{f}, \lambda_{n}^{f}, \lambda_{m}^{f}\right\}$. However, due to the fact that the total number of electrons remains constant when the pressure or magnetic field is applied, we need to satisfy equation for the chemical potential $\mu$ via the condition

$$
n=\frac{1}{\Lambda} \sum_{\mathbf{k} b \sigma} f\left(E_{\mathbf{k} \sigma}^{b}\right),
$$

with $f$ being the Fermi distribution. The equilibrium thermodynamic potential functional defines also the ground state energy, $E_{G}=\left.\mathcal{F}\right|_{0}+\Lambda \mu_{0} n$, where subscript ${ }^{\prime} 0^{\prime}$ denotes the optimal values. After carrying out the minimization, we can also calculate total spin polarization from

$$
m \equiv m_{c}+m_{f}=\frac{1}{\Lambda} \sum_{\mathbf{k} b \sigma} \sigma f\left(E_{\mathbf{k} \sigma}^{b}\right) .
$$

The numerical calculations with the precision of at least $10^{-7}$ were carried out for a two dimensional, square lattice of $\Lambda=512 \times 512$ size, and for low temperatures $\beta \equiv 1 / k_{B} T \geqslant 1500$, emulating the $T \rightarrow 0$ limit.

Results. First, we analyze FM and PM solutions in the field absence. In Figure 1 we draw phase diagram on total filling-hybridization strength plane. For low hybridization, FM phases are favored due to the negative balance between increase of the kinetic and decrease of the Coulomb energies, caused by a relative shift of the spin-resolved DOS. This is visualized by the diminution of the spin-subband overlap up to the FS - cf. Figs. 1 $1 a$ and $1 \mathrm{~b}$. The appearance of a spontaneous polarization, as a result of a competition between the kinetic and the Coulomb energies, is in fact the feature of the Stoner mechanism for the band FM onset. In comparison to the usual single-band (e.g. Hubbard) model, we can distinguish in a natural manner between the two FM phases. The first (FM1) appears when the chemical potential is placed in the hybridization gap, between the spin subbands of the lower hybridized band which is characterized also by the magnetization equal to $m=2-n$ (cf. Fig. [1b). In that situation, only the spin-minority carriers are present at and near FS. The second phase (FM2) emerges when we further lower the hybridization and thus the chemical potential enters the majority spinsubband DOS (cf. Fig. 1 $1 a$ ), giving rise to a step (discontinuous) increase in magnetization (cf. Fig. 2a). In the limit of strong hybridization, for a fixed total filling, when the correlations weaken due to lowering of $f$-orbital average occupancy $\left(n_{f} \lesssim 0.85\right.$, c.f. Fig. 2 c), the kinetic energy gain outbalances a subsequent reduction of the average Coulomb interaction and PM phase is energetically favorable. Similar mechanism for the formation of FM and in particular, characterization of phases, was studied before in Refs. 2326.

We presume that the main effect of the pressure exerted on the material can be modeled by a concomitant strengthening of the hybridization amplitude. Thus, 

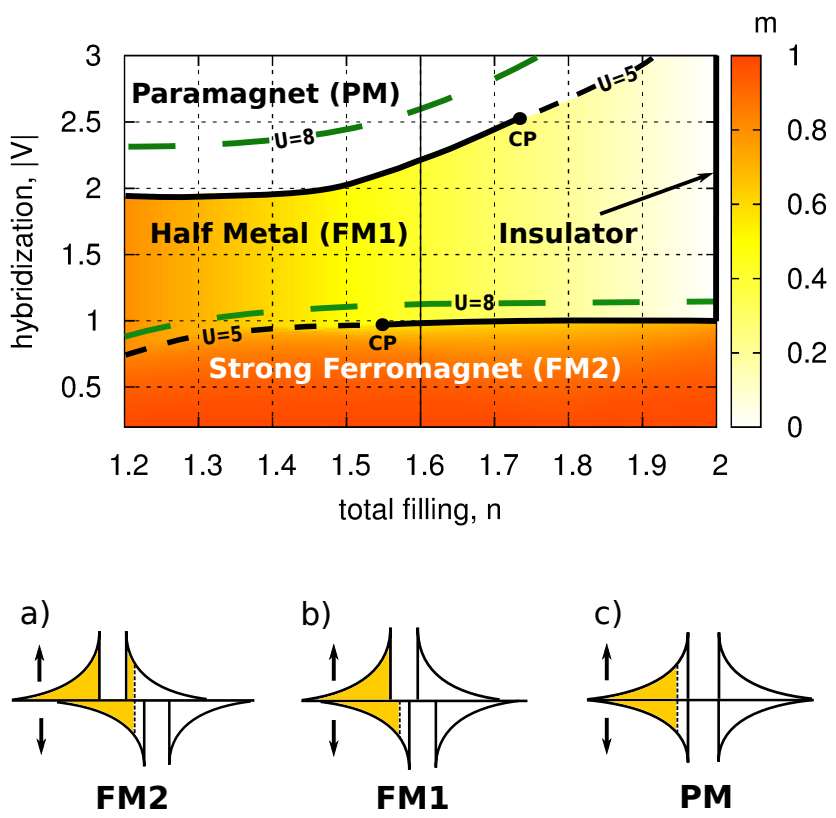

FIG. 1. (Color online): (top) Phase diagram on plane total filling-hybridization strength for the zero field, containing both FM and PM phases for $U=5$. Color scale denotes total spin polarization, $m$. Phases are divided by the dashed and the solid lines. Dashed lines denote the 2nd order transition, whereas the solid - the 1st order transition with the critical points, CP. Fine-dashed lines mark how the phase borders would change for $U=8$. (a-c) Figures a-c depict a schematic spin-resolved density of states corresponding to the phase sequence appearing along the solid vertical line (from bottom to top).

from Fig. 1 it can be seen that for the total filling $n$ in the range $1.55-1.75$, the sequence of phases and the order of the transitions are the same, as those found experimentally for $\mathrm{UGe}_{2}$ by increasing the pressure ${ }^{4.8}$. As a representative band filling we have selected $n=1.6$ marked by the vertical line in Fig. 1. In fact, as we compare the magnetization versus hybridization along the traced line (cf. Fig. $2 a$ ) with the corresponding experimental data ${ }^{4}$ (cf. Fig. $2 b$ ) we find good qualitative resemblance. Moreover, the magnetization differentiation among the orbitals (cf. Fig. $2 a a)$ is in an agreement with the neutron scattering data 10,11 at ambient pressure (in our model $|V| \simeq 0.5$ ), where it was found that almost exclusively electrons from uranium atoms ( $f$-orbital) contribute to the ferromagnetism. In our Stoner-like picture it is resulting from the fact that the competition between Coulomb repulsion and hybridization-induced itineracy concerns mainly the $f$ electrons. Furthermore, as for low hybridization (in FM2 phase) we obtain a small compensating polarization due to the $c$-electrons, we suggest that the experimentally observed small negative magnetization between the uranium atoms at ambient pressure $\underline{\underline{11}}$ may come from the delocalized cloud of conduction electrons.
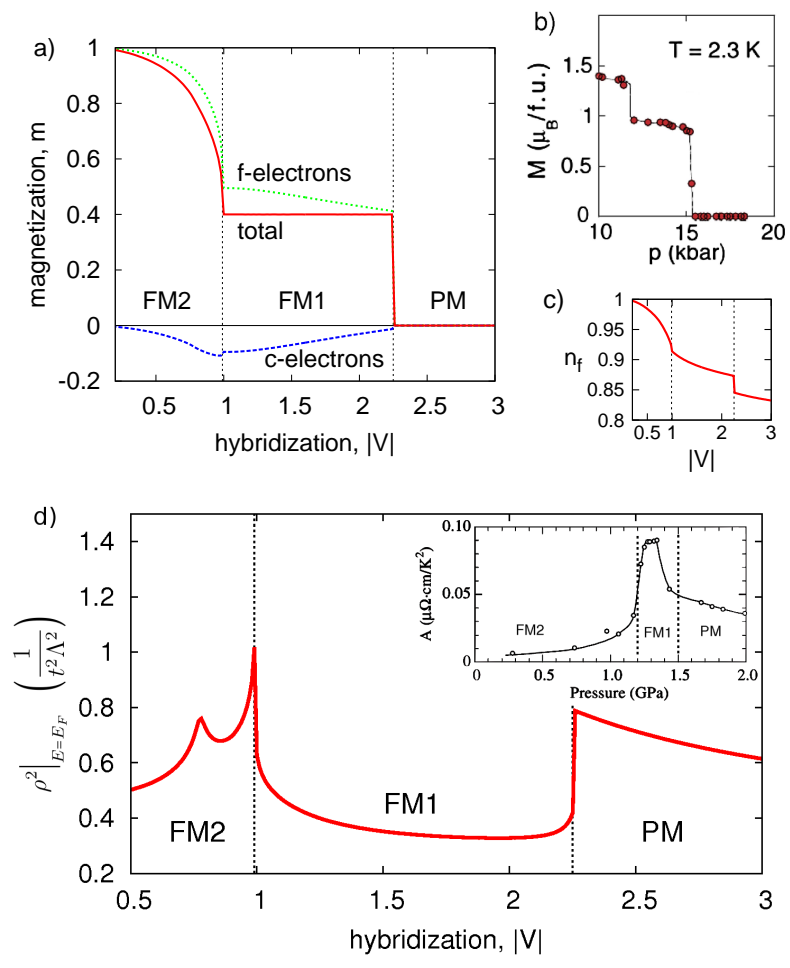

FIG. 2. (Color online): (a) Magnetization as a function of hybridization strength for the band filling $n=1.6$, and the Coulomb repulsion $U=5$. Both phase transitions induced by the hybridization change are of the 1st order. (b) Corresponding experimental results from Ref. 4. (c) $f$-orbital filling as a function of hybridization. (d) Square of DOS at the Fermi level versus $|V|$ through the phase sequence. Inset: Experimentally measured $T^{2}$-term coefficient $A$ of the resistivity versus pressure from Ref. 18.

Our microscopic description of the phase transitions induced by the change of the FS topology compares also favorably with the electronic-state features of $\mathrm{UGe}_{2}$ derived from de Haas van Alphen oscillations 18.19. In Ref. 18 it is suggested, that the majority spin FS disappears in the FM1 phase, in complete accord with the character of DOS presented in the Fig. $1 \mathrm{~b}$. We also reproduce the feature of an abrupt change of the FS at the FM1-PM

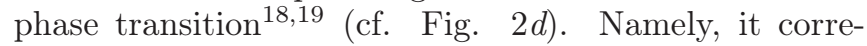
sponds here to the step change of the chemical potential position merging into both bands. Furthermore, in the experimental data at the metamagnetic phase transition there is observed significant enhancement of the quasiparticle mass renormalization $\frac{19}{}$. As it is proportional to the DOS at Fermi level, in Fig. $2 d$ we provide the corresponding behavior, which can be understood within our model by the chemical potential crossing high hybridization peak in the majority spin subband. The transition leads then to a step change of FS only in the majority spin subband, while the minority subband evolves rather continuously, what is also seen experimentally $\underline{19}$.

For the sake of completeness, we have shown in the inset in Fig. $22 d$ the pressure dependence of the $T^{2}$ term 

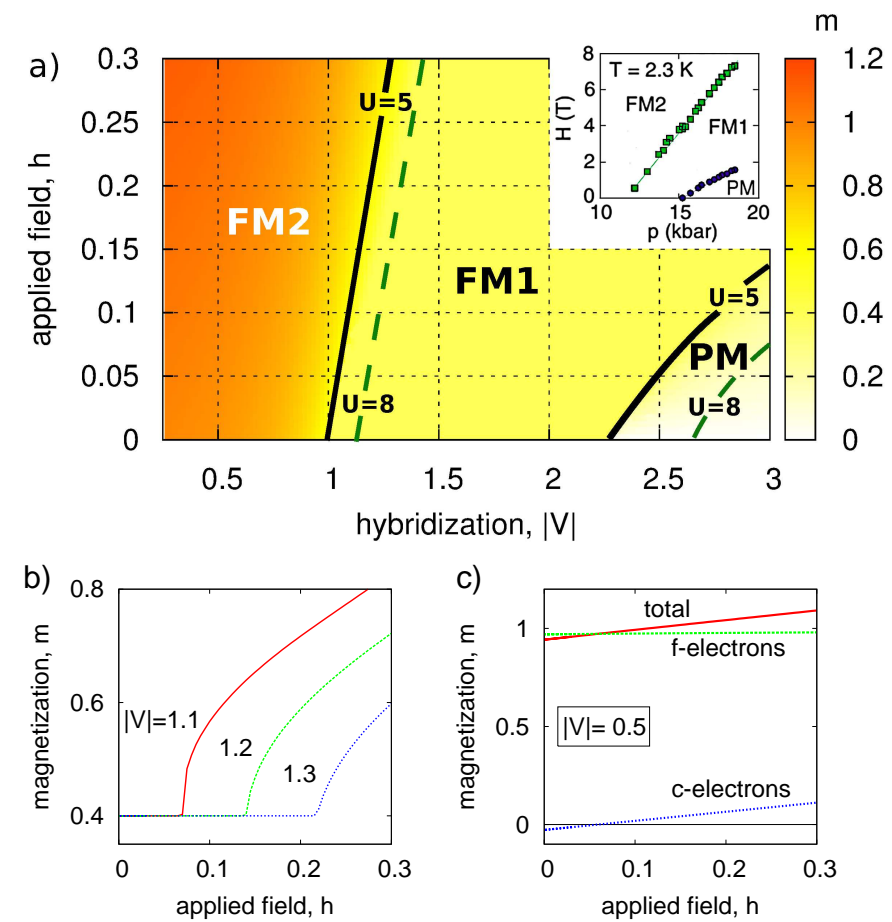

FIG. 3. (Color online): (a) Phase diagram on the applied field-hybridization strength plane for $n=1.6$ and $U=5$. Color scale denotes total spin polarization. The dashed lines mark the phase stability thresholds for $U=8$. In the inset we show experimental results $\stackrel{4}{*}$. (b) Magnetization versus applied field for selected hybridization strengths when system is entering into FM1 to FM2 phase-transition regime. (c) Evolution of orbital-resolved magnetization with the field for low hybridization, $|V|=0.5$ (mimicking ambient pressure). Note the very small $c$-electron polarization up to $h \simeq 0.1$.

of resistivity $\underline{18}$ as it should have roughly the same dependence as squared DOS at the FS, versus $|V|$ (we assume that the Kadowaki-Woods scaling holds). However, the jump that we obtain at the FM1 - PM transition has not been observed in the resistivity measurements 18 .

In the applied field, our model is also in good agreement with available experimental data for $\mathrm{UGe}_{2}$. In Figure $3 a$ we display phase diagram on hybridizationapplied-magnetic-field plane that corresponds to that determined experimentally $\underline{\underline{4}}$ (cf. Fig. $3 a$ inset). Similarly as in Ref. 4, the magnetization at the phase transition between FM1 and FM2 triggered by the applied magnetic field, starts from the same baseline, independently of the hybridization strength (cf. Fig. 3b). However, one should note that, due to the fact that pressure changes not only the hybridization magnitude, but also other microscopic parameters, we are not able to reproduce the magnetization cascade with the increasing magnetic field when crossing the transitions.

The next feature found in $\mathrm{UGe}_{2}$ at ambient pressure is an initial lack of measurable polarization on the germanium atoms with the increasing magnetic field, as inferred from the neutron scattering data 10 . In our model we find a similar trend. For low hybridization $(|V| \simeq 0.5$ emulating ambient pressure), $c$-electrons polarization increases slowly, and even up to $h \approx 0.1$ it is negligible (cf. Fig. 3c).

Remarks. With the simple but powerful technique based on the generalized Gutzwiller ansatz (SGA method), applied to the Anderson lattice model, we have constructed a microscopic model of FM in $\mathrm{UGe}_{2}$. Namely, we are able to reproduce main experimental features observed at low temperature, by applying either pressure or magnetic field (cf. Figs. 2 $2 a$ and $3 a$ ). FM properties can be rationalized within the simplest hybridized two-orbital model, without taking into account the $f$-orbital degeneracy, i.e., by effectively incorporating both the Coulomb and the Hund's-rule interaction into an effective interaction $U$, as would be also the case in the Hartree-Fock approximation $\underline{?}$.

To determine the stability of SC inside FM phase, the present approach should be extended to account for the Hund's-rule interaction explicitly what can be crucial for a formation of the unconventional triplet $\mathrm{SC} 6 \mathbf{6}, 27,28$. If this is the case, it can be triggered even by a purely repulsive Coulomb interaction in conjunction with the residual Hund's rule coupling, as discussed in Refs. 27 and 28. This issue requires a separate analysis. Another path for discussing the coexistence of SC with FM could be going beyond the Gutzwiller approximation, where we account also for the more distant correlations when determining the effective Hamiltonian ${ }^{29,30}$. Here, the central question is whether the spin triplet pairing should be treated on the same footing as ferromagnetism, i.e., appears already in direct space formulation $-6,7,27,28$ or is it mediated by collective spin fluctuations in ferromagnetic phase $14,31-33$ among already well defined quasiparticles. A crossover from the latter to the former approach is expected to take place with the increasing strength of the repulsive Coulomb interaction U.

Acknowledgements. The work has been partially supported by the Foundation for Polish Science (FNP) under the Grant TEAM, as well as by the National Science Centre (NCN) under the Grant MAESTRO, No. DEC-2012/04/A/ST3/00342. We would like to thank J. Kaczmarczyk for discussions and critical reading of the manuscript.
* marcin.wysokinski@uj.edu.pl

$\dagger$ marcin.abram@uj.edu.pl

ufspalek@if.uj.edu.pl

1 S. S. Saxena, P. Agarwal, K. Ahilan, F. M. Grosche,
R. K. W. Haselwimmer, M. J. Steiner, E. Pugh, I. R. Walker, S. R. Julian, P. Monthoux, G. G. Lonzarich, A. Huxley, I. Sheikin, D. Braithwaite, and J. Flouquet, Nature 406, 587 (2000) 
2 A. J. Leggett, Rev. Mod. Phys. 47, 331 (1975)

3 J. F. Annett, B. L. Györffy, and K. I. Wysokiński, New J. Phys. 11, 055063 (2009)K. I. Wysokiński, J. F. Annett, and B. L. Györffy, Phys. Rev. Lett. 108, 077004 (2012)M. Gradhand, K. I. Wysokiński, J. F. Annett, and B. L. Györffy, Phys. Rev. B 88, 094504 (2013)

${ }^{4}$ C. Pfleiderer and A. D. Huxley, Phys. Rev. Lett. 89, 147005 (2002)

5 V. Taufour, D. Aoki, G. Knebel, and J. Flouquet, Phys. Rev. Lett. 105, 217201 (2010)

6 J. Spałek, Phys. Rev. B 63, 104513 (2001)

7 M. Zegrodnik and J. Spałek, Phys. Rev. B 86, 014505 (2012)

8 C. Pfleiderer, Rev. Mod. Phys. 81, 1551 (2009), (chapter III. A)

9 D. Aoki and J. Flouquet, J. Phys. Soc. Jpn. 81, 011003 (2012)

10 A. Huxley, I. Sheikin, E. Ressouche, N. Kernavanois, D. Braithwaite, R. Calemczuk, and J. Flouquet, Phys. Rev. B 63, 144519 (2001)

11 N. Kernavanois, B. Grenier, A. Huxley, E. Ressouche, J. P. Sanchez, and J. Flouquet, Phys. Rev. B 64, 174509 (2001)

12 K. Hirohashi and K. Ueda, J. Phys. Soc. Jpn. 73, 1576 (2004)

13 D. Belitz, T. R. Kirkpatrick, and J. Rollbühler, Phys. Rev. Lett. 94, 247205 (2005)

14 K. G. Sandeman, G. G. Lonzarich, and A. J. Schofield, Phys. Rev. Lett. 90, 167005 (2003)

15 A. B. Shick and W. E. Pickett, Phys. Rev. Lett. 86, 300 (2001)

16 V. H. Tran, S. Paschen, R. Troć, M. Baenitz, and F. Steglich, Phys. Rev. B 69, 195314 (2004)

17 Y. Onuki, I. Ukon, S. Won Yun, I. Umehara, K. Satoh, T. Fukuhara, H. Sato, S. Takayanagi, M. Shikama, and A. Ochiai, J. Phys. Soc. Jpn. 61, 293 (1992)

18 R. Settai, M. Nakashima, S. Araki, Y. Haga, T. C. Kobayashi, N. Tateiwa, H. Yamagami, and Y. Onuki,
J. Phys: Condens. Matter 14, L29 (2002)

19 T. Terashima, T. Matsumoto, C. Terakura, S. Uji, N. Kimura, M. Endo, T. Komatsubara, and H. Aoki, Phys. Rev. Lett. 87, 166401 (2001)

20 D. Vollhardt, Rev. Mod. Phys. 56, 99 (1984)

21 T. M. Rice and K. Ueda, Phys. Rev. Lett. 55, 995 (1985)

22 P. Fazekas and B. H. Brandow, Phys. Scr. 36, 809 (1987)

23 Approach derived in J. Jȩdrak, J. Kaczmarczyk, and J. Spałek, arXiv:1008.0021J. Jędrak and J. Spałek, Phys. Rev. B 81, 073108 (2010)83, 104512 (2011)J. Kaczmarczyk and J. Spałek, 84, 125140 (2011)M. Wysokiński, J. Jȩdrak, J. Kaczmarczyk, and J. Spałek, AIP Conf. Proc. 1485, 319 (2012)O. Howczak and J. Spałek, J. Phys: Condens. Matter 24, 205602 (2012)M. Abram, J. Kaczmarczyk, J. Jędrak, and J. Spałek, Phys. Rev. B 88, 094502 (2013)O. Howczak, J. Kaczmarczyk, and J. Spałek, Phys. Stat. Solidi (b) 250, 609 (2013)M. M. Wysokiński and J. Spałek, J. Phys.: Condens. Matter 26, 055601 (2014)

24 R. Doradziński and J. Spałek, Phys. Rev. B 56, R14239 (1997)

25 R. Doradziński and J. Spałek, Phys. Rev. B 58, 3293 (1998)

${ }^{26}$ K. Kubo, Phys. Rev. B 87, 195127 (2013)

27 M. Zegrodnik, J. Spałek, and J. Bünemann, New J. Phys. 15, 073050 (2013)

28 M. Zegrodnik, J. Bünemann, and J. Spałek, New J. Phys. 16, 033001 (2014)

29 J. Kaczmarczyk, J. Spałek, T. Schickling, and J. Bünemann, Phys. Rev. B 88, 115127 (2013)

30 J. Kaczmarczyk, J. Bünemann, and J. Spałek, New J. Phys. 16, 073018 (2014)

31 D. Fay and J. Appel, Phys. Rev. B 22, 3173 (1980)

32 T. R. Kirkpatrick, D. Belitz, T. Vojta, and R. Narayanan, Phys. Rev. Lett. 87, 127003 (2001)

33 R. Roussev and A. J. Millis, Phys. Rev. B 63, 140504 (2001) 